\title{
Factors Limiting Buprenorphine Prescriptions in Sacramento and EI Dorado County
}

\author{
Alana Freifeld ${ }^{1}$, Neeraj Ramakrishnan ${ }^{1}$, Jose Puglisi ${ }^{1}$, Aimee Moulin ${ }^{2}$, Joshua Elder ${ }^{2,}$ * \\ ${ }^{1}$ College of Medicine, California Northstate University, Elk Grove, USA \\ ${ }^{2}$ Department of Emergency Medicine, University of California Davis, Sacramento, USA
}

Email address:

jelder@ucdavis.edu (J. Elder)

${ }^{*}$ Corresponding author

\section{To cite this article:}

Alana Freifeld, Neeraj Ramakrishnan, Jose Puglisi, Aimee Moulin, Joshua Elder. Factors Limiting Buprenorphine Prescriptions in Sacramento and El Dorado County. American Journal of Internal Medicine. Vol. 7, No. 3, 2019, pp. 66-71. doi: 10.11648/j.ajim.20190703.13

Received: April 8, 2019; Accepted: May 27, 2019; Published: June 26, 2019

\begin{abstract}
Buprenorphine is an effective treatment for opioid use disorder (OUD). Despite the demonstrated efficacy of Medication Assisted Treatment (MAT), few healthcare providers are licensed to prescribe Buprenorphine for OUD. The purpose of this study was to assess the attitudes and prescribing practices of providers licensed to treat patients Buprenorphine in two counties in Northern California. We aim to identify barriers to prescribing Buprenorphine for OUD. This is a descriptive study to understand provider practices, attitudes, and barriers towards Buprenorphine prescribing. Of the $95 \mathrm{x}$-waivered providers identified in Sacramento and El Dorado County, 36 (38\%) responded to our phone survey. 12 (33\%) were women, and the three most common specialties were Family Medicine 19 (53\%), Addiction Medicine 8 (22\%), and Psychiatry 8 (22\%). Of the survey participants, $33(92 \%)$ actively prescribe Buprenorphine. Self-motivation was cited as the most common reason to become $\mathrm{x}$-waivered $29(81 \%)$, while the least cited reason was an institutional requirement $3(8 \%)$. The most common motivations for prescribing Buprenorphine included low overdose potential 30 (91\%), drug efficacy 28 (85\%), and low abuse potential $27(82 \%)$. The greatest barriers to Buprenorphine prescribing included poor reimbursement 15 (46\%), time constraints $13(39 \%)$, and patient preference for opiates 11 (33\%). Overall, most of the interviewed providers $30(83 \%)$ believed Buprenorphine should be prescribed more frequently to manage addiction or pain. This study found that the majority of X-waivered providers in Sacramento and El Dorado Counties do prescribe Buprenorphine but not at their full capacity.
\end{abstract}

Keywords: Buprenorphine, X-waiver, Opioid Epidemic, Addiction, Dependency

\section{Introduction}

The increasing number of opioid related overdose and deaths in the United States has garnered national attention. In 2016 alone there were over 42,000 overdose deaths in the United States from opioids [1]. Similarly, opioid overdose and mortality have significantly impacted the local communities of Sacramento County and El Dorado County. Between 2012 and 2014, the number of opioid related deaths per 100,000 in Sacramento and El Dorado Counties were 698 and 115 respectively [2]. Given the magnitude of this crisis, accessible and safe treatment options for opioid use dependency is of great importance.

Buprenorphine is a partial $\mu$-opioid agonist and $\kappa$-opioid antagonist approved for clinical use in October 2002 to help patients quit or reduce opioid dependence. Unlike other addiction treatments such as Methadone, Buprenorphine can be prescribed in any outpatient setting by a provider with an additional $\mathrm{x}$-waiver attached to their DEA license. The $\mathrm{x}$ waiver training involves an eight hour course for MDs and a 24 hour course for NPs and Pas [3]. Buprenorphine is a safe, effective treatment for OUD with low risk of overdose and respiratory depression [4]. Despite the existence of this powerful opioid cessation tool, relatively few providers have completed the course necessary to obtain the waiver to prescribe Buprenorphine. Our study identified 95 registered Buprenorphine providers (medical doctors, nurse practitioners, and medical assistants) for a population of over 
1.68 million people in Sacramento and El Dorado County.

The barriers to prescribing Buprenorphine and attitudes towards the use of Buprenorphine for OUD in Sacramento and El Dorado Counties have not previously been described. This study aimed to better understand prescribing practices and obstacles faced by $\mathrm{x}$-waivered physicians in this area. The goal of this study is to better characterize the regional landscape so as to inform community programs and public policy with the intention to increase awareness of and access to Buprenorphine for OUD.

\section{Methods}

\subsection{Participants and Inclusion Procedure}

We identified $87 \mathrm{x}$-waivered providers registered with the Substance Abuse and Mental Health Services Administration (SAMHSA) in Sacramento and El Dorado Counties. SAMHSA provides a public database of physicians, physician assistants, and nurse practitioners with an x-waiver license. An additional eight eligible providers were also solicited through referrals from interviewed participants. Exclusion criteria for participants included practice outside of the Sacramento/El Dorado Counties and/or inactive xwaivered license. Of the 95 providers identified by the study, 36 providers $(38 \%)$ participated in the study.

\subsection{Design}

The 19 question survey was developed through a literature review and interviews with a local expert panel of addiction medicine specialists. The survey consisted of questions regarding basic demographic information, current prescription patterns, drug satisfaction, and barriers to prescription of Buprenorphine. Multiple choice questions, five point satisfaction scales, and fill-in-the-blank sections were utilized. Conditional branching was used to create two separate survey paths depending on whether or not participants currently prescribe Buprenorphine. The survey was then validated through interviews with the local expert panel prior to administration.

\subsection{Procedures and Measures}

The survey was conducted August 2017 to December 2017 via a combination of phone calls and email invitations. Email invitations were sent to providers upon request based on provider preference. Phone numbers were acquired from the public SAMHSA list, while email addresses were acquired directly from study participants who wished to complete the survey through email rather than phone. Participants were consented by phone or email in conjunction with the survey. Participants were contacted up to 3 times by phone and/or contacted up to 3 times via email. One additional follow-up question regarding Buprenorphine prescription patterns was also emailed in Feb 2018 to participants who had completed the survey.
$\mathrm{X}$-waivered names collected from SAHMSA

(87)

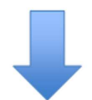

$\mathrm{X}$-waivered providers called and interviewed via phone or sent an email with direct survey link by request (95)

Referral to unlisted $\mathrm{x}$ waivered providers from interviewees (8)

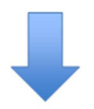

Patients who did not answer were called two additional times (59)

Figure 1. How X-Waivered Provider's Were Enrolled in Study.

\subsection{Data Analysis}

Phone responses from participants were anonymously recorded into an online survey tool. Data from phone responses and email surveys were exported and descriptively analyzed with the Prism ${ }^{\circledR}$ program Version 7.03 from GraphPad Software Inc as well as STATA. Emails contained a direct link to the blank online survey.

This protocol was approved by the Institutional Review Board of California Northstate University College of Medicine.

\section{Results}

\subsection{Demographics}

Survey data was collected by way of a telephone survey or email interview. 36 providers out of a possible 95 (38\%) responded to the request for survey. The survey sample represented a cross section of physicians in the Sacramento and El Dorado Counties who are registered x-waivered providers. The three most common respondent specialties included family medicine $19(53 \%)$, addiction medicine 8 $(22 \%)$, and psychiatry $8(22 \%)$ (Table 1$)$. A total of nine professional specialties were reported, and multiple respondents had more than one professional specialty. Years in practice ranged from 0 (new physician) to more than 20 years, with the majority having more than 20 years of experience. The majority of respondents $24(67 \%)$ were males (Table 1).

Table 1. Specialties, Years in Practice, and Gender, among X-waivered Physicians Surveyed.

\begin{tabular}{lll}
\hline Participants (N) & $\mathbf{3 6}$ & $\mathbf{\%}$ \\
\hline Professional Specialty (N) & & \\
Family Medicine & 19 & $53 \%$ \\
Internal Medicine & 5 & $14 \%$ \\
Psychiatry & 8 & $20 \%$ \\
Addiction Medicine & 8 & $20 \%$ \\
Emergency Medicine & 1 & $2.5 \%$ \\
Neurology & 1 & $2.5 \%$ \\
Occupational Medicine & 1 & $2.5 \%$ \\
Pediatrics & 1 & $2.5 \%$ \\
\hline
\end{tabular}




\begin{tabular}{lll}
\hline Participants (N) & $\mathbf{3 6}$ & $\mathbf{\%}$ \\
\hline Aerospace & 1 & $2.5 \%$ \\
*Some providers had multiple specialties & & \\
Years in Practice (N) & & \\
$0-5$ & 10 & $27 \%$ \\
$6-10$ & 4 & $11 \%$ \\
$11-15$ & 5 & $14 \%$ \\
$16-20$ & 2 & $6 \%$ \\
$>20$ & 15 & $42 \%$ \\
Sex (N) & & \\
Male & 24 & $67 \%$ \\
Female & 12 & $33 \%$ \\
\hline
\end{tabular}

\subsection{Motivations to Become $X$-waivered and Uses of} Buprenorphine

The most commonly cited motivations to become $\mathrm{x}$ -

a)

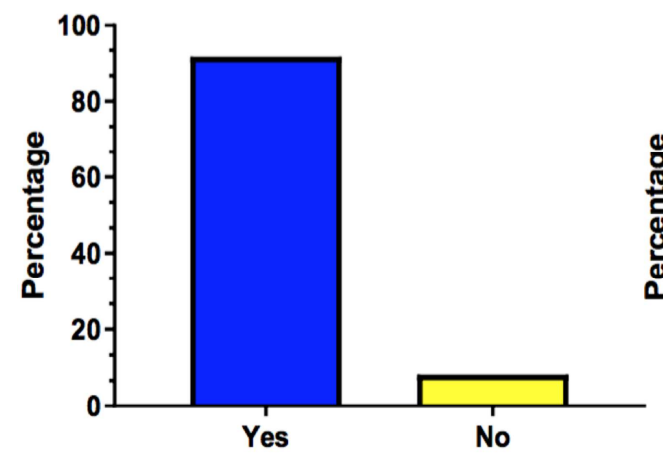

waivered included self-motivation $29(81 \%)$, continued medical education $12(33 \%)$, and peer influence $11(31 \%)$. Of the 33 prescribers, the most important factors that impacted provider readiness to prescribe Buprenorphine included efficacy 28 (85\%), low overdose potential $30(91 \%)$, and low abuse potential $27(81 \%)$.

Of the thirty-six respondents interviewed, thirty-three $(92 \%)$ reported that they use Buprenorphine to treat patients who are opioid dependent or for pain (figure 4). Of the thirtythree Buprenorphine providers 13 (39\%) prescribe Buprenorphine for opioid dependency only, while 20 (60\%) use Buprenorphine to manage both opioid dependency and pain. Zero survey respondents used Buprenorphine for pain only.

b)

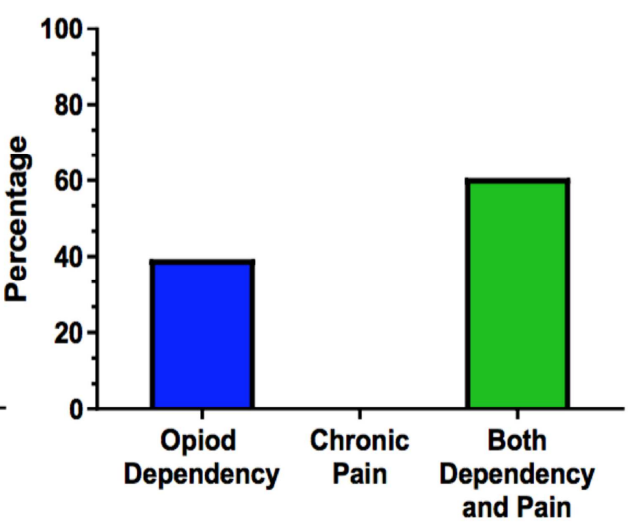

Figure 2. Prescription Practices of Buprenorphine.

Percentage of $\mathrm{x}$-waivered providers prescribing Buprenorphine within their practice (figure 2a) and percentage of $\mathrm{x}$-waivered providers prescribing Buprenorphine for opioid dependency, chronic pain, or both (figure 2b).

\subsection{Barriers to Prescribing Buprenorphine}

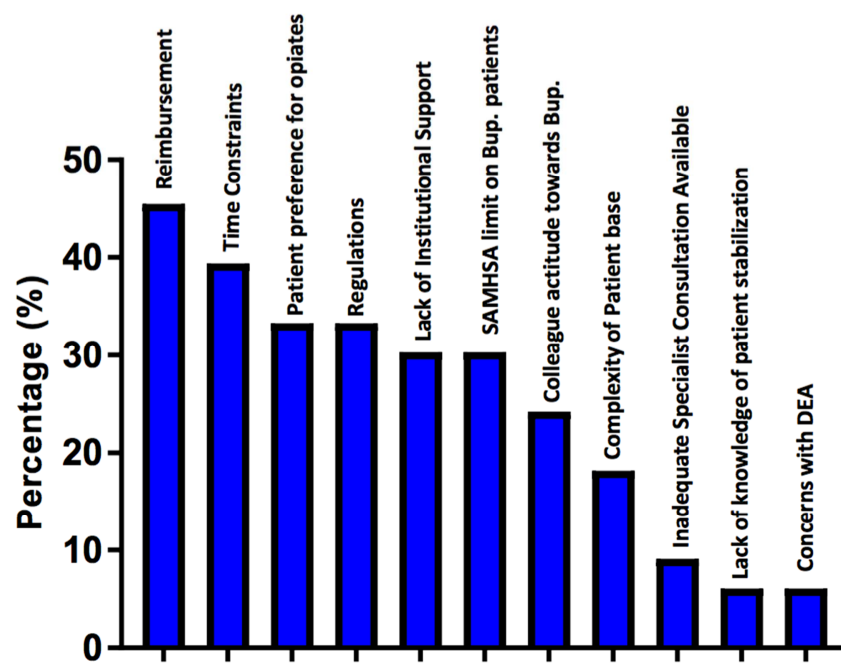

Figure 3. Barriers to prescribing Buprenorphine to Eligible Patients ( $n=33$ ).
Figure 3 displays the barriers that surveyed providers reported to prescribing Buprenorphine. Reimbursements were cited most frequently as a barrier to Buprenorphine prescription $15(45 \%)$. This was closely followed by time constraints $13(39 \%)$, patient preference for opiates $11(33 \%)$, lack of institutional support $10(30 \%)$, and SAMHSA waiver limit on number of patients $10(30 \%)$. The least frequently cited barriers were concerns with the DEA 2 (6\%), inadequate specialist consultation $2(6 \%)$, and lack of knowledge of patient stabilization $3(9 \%) .2$ (6\%) providers reported no barriers.

Most commonly cited barriers to prescribing Buprenorphine by percentage of respondents endorsing each barrier.

\section{4. \% of Eligible Patient Populations Receiving Buprenorphine Prescriptions}

Of the 33 Buprenorphine prescribers interviewed, 10 providers $(30 \%)$ prescribe Buprenorphine to $100 \%$ of their eligible patient populations, 25 providers (76\%) prescribe Buprenorphine to $50 \%$ or more of their patients who are candidates to receive, while 4 providers $(12 \%)$ had patient populations that were candidates for Buprenorphine, but did not prescribe the drug (figure 4). After taking an eight hour 
course for the x-waiver license, providers can initially be approved for a 30-patient limit on their waiver. Those who wish to prescribe to more than 30 patients may apply to SAMHSA to authorize an increase of their waiver limits to 100 patients. Under new federal regulations, those who have prescribed buprenorphine to 100 patients for at least one year can apply to increase their patient limits to 275 patients.

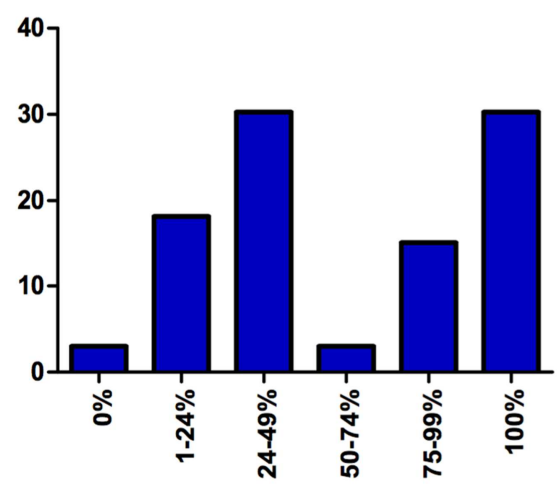

Figure 4. Percentage of Eligible Patient Populations Receiving Buprenorphine Prescriptions.

Survey respondents were asked to estimate the percentage of eligible patient populations within their practice who received Buprenorphine prescriptions. Reported as percentage of respondents who prescribe Buprenorphine to $\mathrm{X} \%$ of their eligible population.

\subsection{Buprenorphine Waiver Capacity by Practice}

Four weeks after the initial survey collection period, the thirty-six participants were contacted again via telephone to ask two follow-up questions. Of the thirty-six initial respondents, twelve completed the two follow-up survey questions. From this follow-up, it was determined that ten of the twelve providers are part of a group practice (83\%), and the majority of providers are not using the Buprenorphine waiver to full capacity (Table 2). Of these twelve respondents, 8 of them $(67 \%)$ had applied to increase their waiver beyond the standard 30-patient waiver. Two of the Buprenorphine providers practice in a group setting where they pooled their waivers in order to prescribe to and manage more than 275 patients receiving Buprenorphine.

Table 2. Buprenorphine Waiver Capacity by Practice $(n=12)$.

\begin{tabular}{lll}
\hline $\begin{array}{l}\text { Type of } \\
\text { Practice }\end{array}$ & $\begin{array}{l}\text { Buprenorphine Waiver } \\
\text { Capacity }\end{array}$ & $\begin{array}{l}\text { Current Number of } \\
\text { Patients on Waiver }\end{array}$ \\
\hline Group & 30 & 0 \\
Group & 100 & 1 \\
Group & 275 & 12 \\
Group & 280 & 210 \\
Group & 30 & 4 \\
Group & 100 & 16 \\
Group & 30 & 10 \\
Group & 30 & 2 \\
Group & 100 & 60 \\
Group & 430 & 196 \\
Solo & 275 & 45 \\
Solo & 100 & 58 \\
\hline
\end{tabular}

\section{Discussion}

This study assessed the attitudes and barriers among $\mathrm{x}$ waivered providers towards prescribing Buprenorphine in El Dorado and Sacramento County. This study further characterizes factors limiting Buprenorphine prescriptions in the Sacramento metro region. The overwhelming majority of the $\mathrm{x}$-waivered providers interviewed in our study prescribed Buprenorphine 33 $(92 \%)$. In addition to the high proportion of addiction medicine $8(22 \%)$ and psychiatry $8(22 \%)$ specialists, the majority of interviewed Buprenorphine prescribers were family medicine practitioners 19 (53\%). Previous studies conducted in Washington state and New York also illustrated significant involvement of primary care fields such as Internal Medicine and Family Medicine in Buprenorphine prescribing [5-6]. Our sample included twice as many male compared to female providers. This ratio of males to female providers is also present on the $\mathrm{x}$-waivered provider list on the SAMHSA website.

The majority of $\mathrm{x}$-waivered providers in our study either had 5 or less years of experience $10(27 \%)$ or had 20 or more years of experience $15(42 \%)$. This trend in years of experience was similar to a descriptive study conducted in New York regarding Buprenorphine prescription patterns. Kermack et al illustrated $36 \%$ of interviewed $\mathrm{x}$-waivered providers had less than 5 years of experience, while $32 \%$ had $20+$ years of experience [5]. Self-motivation 29 (81\%) was the most frequently cited reason in our study for obtaining an $\mathrm{x}$-waiver license. More specific reasons included catering to a medically underserved and stigmatized population of patients suffering from substance abuse, lessening the severity of the opioid crisis in the United States, and having a personal history of working with opioid dependent patients. Other major motivating factors included continued medical education $12(31 \%)$ and peer influence $11(31 \%)$. There were no differences in motivating factors between age distributions. There is a scarcity of other studies exploring why providers specifically pursue a x-waiver. Characterizing the motivations of these providers could shed light on populations that are more likely to become $\mathrm{x}$-waivered.

Top cited motivating factors for prescribing Buprenorphine were low overdose potential $30(91 \%)$, efficacy $28(85 \%)$, and low abuse potential 27 (81\%). Providers consistently characterized Buprenorphine as safe and effective. 13 of the providers $(39 \%)$ in this study used Buprenorphine for solely treating opioid dependency while 2 providers $(60 \%)$ used Buprenorphine to manage both opioid dependency and pain. Off-label use of Buprenorphine to treat chronic pain has increased in recent years [7]. Buprenorphine has been shown to both reduce pain and improve the quality of life for this population [8]. However, little is known surrounding clinician behavior and attitude towards Buprenorphine for the management of chronic pain. A 2014 study from Rosen et al. attempted to characterize prescription practices and attitudes towards Buprenorphine for chronic pain management. In their study, Sublingual Buprenorphine for chronic pain a survey of clinician prescribing practices, the group collected a 36 -item survey from 230 clinicians across the country to 
assess attitudes and practices regarding prescription of sublingual Buprenorphine and other opioids. The group concluded that sublingual Buprenorphine is indeed being used to treat chronic pain, but the context for when it is appropriate to prescribe it for pain management remains unclear [9]. Chronic pain patients face the challenges of becoming prescribed opioid medications that are highly subject to addiction and abuse. Moreover, these patients often require alternative treatments for their pain because of opioid tolerance and opioid-induced hyperalgesia [10]. Studies have also shown that Buprenorphine can reverse opioid-induced hyperalgesia and reduce pain through its mechanism as a kreceptor antagonist [11]. Buprenorphine efficacy and safety profile could make it a powerful solution to patients not only suffering from opioid addiction, but also concomitant pain.

Of the 36 interviewed providers in our study, $12(33 \%)$ gave additional information on their Buprenorphine waiver capacity and current number of patients on their waiver. Capacities ranged from 30-430 patients for some group practices, and all providers practiced well under their capacity limit. Prescription Monitoring Program data from Ohio, California, and Maine from January 2010 to April 2015 has also shown that most Buprenorphine waivered providers practice below their waiver capacity [12]. Sacramento County is not fully augmenting the capacity of their current $\mathrm{x}$-waivered clinicians. Though we have explored individual level barriers, it is important to identify systemic barriers such as the legal system, pharmaceutical industry, societal stigma, and local epidemiologic data regarding OUD.

There is a major shortage of physicians using Buprenorphine to treat OUD in the United States. In fact, 96\% of states report higher rates of opioid abuse/dependence compared to Buprenorphine treatment capacity [13]. Despite its efficacy, there remain barriers to Buprenorphine prescription. In our study, reimbursements 15 (45\%), time constraints $13(39 \%)$, and patient preference for opioids 11 (33\%) were the most frequently cited barriers. Additionally, our study did not find that providers had a lack of knowledge or difficulty in their experience of obtaining a $\mathrm{x}$-waiver. Huhn et al (2017) is a study that quantitatively aimed to understand why Buprenorphine was not being prescribed more frequently for opioid addiction. Hunh found time constraints for additional patients as the highest cited reason to not become $\mathrm{x}$ waivered or prescribe Buprenorphine. Other barriers identified by the study was a lack of knowledge on how to obtain a waiver. Participants also cited information about local counseling resources for providers that supplied them with knowledge for effective prescription of Buprenorphine (40\%) and being paired with an experienced provider (35\%) as being the most important motivators of obtaining an $\mathrm{x}$-waiver [14]. Given the high proportion of $\mathrm{x}$-waivered providers who actively prescribe Buprenorphine in our study, increasing awareness of the $\mathrm{x}$-waiver in the local community could be an avenue to increase Buprenorphine access. Assembling workshops through local community groups such as the local medical society and/or hospital departments and providing these resources can potentially be a novel way to encourage pursuit of X-waivers, while decreasing the impact of barriers to Buprenorphine prescription. Incorporating Buprenorphine treatment into residency training could be another powerful way to increase prescription of this medication [15].

It is also important that particular specialties are more likely to prescribe Buprenorphine and treat OUD. For instance, Walley et al (2008) illustrated that being a primary care physician or solo practitioner were variables that significantly increased odds of prescribing Buprenorphine [16]. OUD is not specific to one area of medicine and increasing efforts need to be made to increase awareness and $\mathrm{x}$-waivered providers in other specialties.

In this study, we identified an additional barrier to Buprenorphine prescriptions: the SAMHSA list of $\mathrm{x}$-waivered providers. When we started this study, the list contained only 76 providers. Their list contained duplicated names, invalid contact information, and providers who had long moved out of the area or passed away. Though the list was eventually updated six months after the start of the study, lapses in accurate information pose a challenge to patients/providers/hospitals seeking $\mathrm{x}$ waivered providers for their needs. More regular updated provider information could reduce this barrier.

As the opioid epidemic has recently begun to garner more national attention, attitudes towards Buprenorphine and its prescription patterns have a higher probability of shifting. In order to increase prescription of this highly effective outpatient drug, emphasis should be placed on educational outreach that recruits more providers to become X-waivered. Dick (2015) et. al asserts that the introduction of the waiver program reduced the percentage of counties with opioid treatment shortages from $98.9 \%$ in 2002 to $46.8 \%$ in 2011 [17]. In addition to increasing knowledge and access of the x-waiver license, community interventions should address notable concerns such as reimbursement, time constraints, and patient preference for opioids. Additionally, the lack of updated regional information regarding Buprenorphine providers and waiver capacity on the SAMHSA website is a barrier to access that can and should be addressed. The potential of Buprenorphine in managing pain through an off-label use can also be further explored as it is already taking place in this community. Increasing the accessibility and awareness of Buprenorphine will equip providers with a powerful MAT to manage OUD.

Limitations to this study include a small sample size $(n=36)$, underrepresentation of $\mathrm{x}$-waivered physicians who do not prescribe Buprenorphine $(n=3)$, and potentially skewed gender demographic in the direction of males. An analysis of our sample was not done to determine if it represented an accurate sample in regard to this skewed gender demographic. The survey also did not address particular themes such as strategies that could increase pursuit of $\mathrm{x}$-waivers or Buprenorphine prescription from the perspective of providers. Inaccuracies from the SAMHSA website for provider contact information posed another limitation. This is a limitation that needs to be explored further, as it is easily possible to opt out of SAMHSA's public database. As a result, there may be many unlisted waiver providers that are not listed on the SAMHSA website. 


\section{Conclusions}

This regional study of Sacramento and El Dorado Counties found that providers with $\mathrm{x}$-waiver training are very likely to prescribe Buprenorphine. However, it was observed less than half of providers prescribe to all of their eligible populations and none use waiver limits to the maximum capacity. The greatest barriers to prescription included reimbursements, time constraints, patient preference for opiates, and regulations. Efforts on the federal and state level to reduce such barriers could improve access to Buprenorphine by both increasing the number of $\mathrm{x}$-waivered providers as well as increasing prescription among those who already prescribe. In the wake of the current opioid epidemic, it is critical that we increase the accessibility of safe and effective MAT such as Buprenorphine. This study brings to focus some of the targets for improving availability of this life-saving medication.

\section{Acknowledgements}

Thank you to the Sierra Sacramento Valley Medical Society for reviewing the SAMHSA list of providers for accuracy.

This research did not receive any specific grant from funding agencies in the public, commercial, or not for-profit sectors.

\section{References}

[1] Opioid Overdose. (2018, October 19). Retrieved January 05, 2019, from https://www.cdc.gov/drugoverdose/

[2] Drug Overdose Deaths. (2018). Retrieved from http://www.countyhealthrankings.org/app/california/2016/mea sure/factors/138/data? sort=sc-0

[3] Quality for NP and PA waivers. (2016, November 21). Qualify for NP and PA Waivers. Retrieved from https://www.samhsa.gov/medication-assistedtreatment/training-materials-resources/qualify-np-pa-waivers

[4] Jones, H. E. (2004). Practical Consideration for the Clinical Use of Buprenorphine. Science \& Practice Perspectives, 2 (2), 4-20. doi:10.1151/spp04224

[5] Kermack, A., Flannery, M., Tofighi, B., Mcneely, J., \& Lee, J. D. (2017). Buprenorphine prescribing practice trends and attitudes among New York providers. Journal of Substance Abuse Treatment, 74, 1-6. doi:10.1016/j.jsat.2016.10.005

[6] Hutchinson, E., Catlin, M., Andrilla, C. H., Baldwin, L., \& Rosenblatt, R. A. (2014). Barriers to Primary Care Physicians Prescribing Buprenorphine. The Annals of Family Medicine, 12(2), 128-133. doi:10.1370/afm.1595

[7] Malinoff, H. L., Barkin, R. L., \& Wilson, G. (2005). Sublingual Buprenorphine Is Effective in the Treatment of Chronic Pain Syndrome. American Journal of Therapeutics, 12(5), 379-384. doi:10.1097/01.mjt.0000160935.62883.ff
[8] Daitch, D., Daitch, J., Novinson, D., Frey, M., Mitnick, C., \& Pergolizzi, J. (2014). Conversion from High-Dose Full-Opioid Agonists to Sublingual Buprenorphine Reduces Pain Scores and Improves Quality of Life for Chronic Pain Patients. Pain Medicine, 15 (12), 2087-2094. doi:10.1111/pme.12520

[9] Rosen, K., Gutierrez, A., Haller, D., \& Potter, J. S. (2014, April). Sublingual Buprenorphine for Chronic Pain. The Clinical Journal of Pain, 30 (4), 295-300. doi:10.1097/ajp.0b013e318298ddad

[10] Chen, K. Y., Chen, L., \& Mao, J. (2014). BuprenorphineNaloxone Therapy in Pain Management. Survey of Anesthesiology, $\quad 58 \quad$ (5), 259-260. doi:10.1097/01.sa.0000452828.37555.a3

[11] Koppert, W., Ihmsen, H., Körber, N., Wehrfritz, A., Sittl, R., Schmelz, M., \& Schüttler, J. (2005, November). Different profiles of buprenorphine-induced analgesia and antihyperalgesia in a human pain model. Pain, 118 (1), 15-22. doi: $10.1016 / j$. pain. 2005.06 .030

[12] Thomas, C. P., Doyle, E., Kreiner, P. W., Jones, C. M., Dubenitz, J., Horan, A., \& Stein, B. D. (2017, December). Prescribing patterns of buprenorphine waivered physicians. Drug and Alcohol Dependence, 181, 213-218. doi:10.1016/j. drugalcdep.2017.10.002

[13] Jones, C. M., Campopiano, M., Baldwin, G., \& Mccance-Katz, E. (2015, August). National and State Treatment Need and Capacity for Opioid Agonist Medication-Assisted Treatment. American Journal of Public Health, 105 (8), 55-63. doi:10.2105/ajph.2015.302664

[14] Huhn, A. S., \& Dunn, K. E. (2017, July). Why aren't physicians prescribing more buprenorphine? Journal of Substance Abuse Treatment, 78, 1-7. doi:10.1016/j. jsat.2017.04.005

[15] Kunins, H. V., Sohler, N. L., Giovanniello, A., Thompson, D., \& Cunningham, C. O. (2013). A Buprenorphine Education and Training Program for Primary Care Residents: Implementation and Evaluation. Substance Abuse, 34 (3), 242-247. doi:10.1080/08897077.2012.752777

[16] Walley, A. Y., Alperen, J. K., Cheng, D. M., Botticelli, M., Castro-Donlan, C., Samet, J. H., \& Alford, D. P. (2008). Office-Based Management of Opioid Dependence with Buprenorphine: Clinical Practices and Barriers. Journal of General Internal Medicine, 23 (9), 1393-1398. doi:10.1007/s11606-008-0686-x

[17] Dick, A. W., Pacula, R. L., Gordon, A. J., Sorbero, M., Burns, R. M., Leslie, D., \& Stein, B. D. (2015). Growth In Buprenorphine Waivers For Physicians Increased Potential Access To Opioid Agonist Treatment, 2002-11. Health Affairs, 34 (6), 1028-1034. doi:10.1377/hlthaff.2014.1205 